\title{
Förster resonance energy transfer as a tool to study photoreceptor biology
}

\author{
Stephanie C. Hovan \\ Case Western Reserve University \\ Department of Ophthalmology and Visual Sciences \\ Cleveland, Ohio 44106
}

\section{Scott Howell}

Case Western Reserve University Visual Sciences Research Center Cleveland, Ohio 44106

Paul S.-H. Park

Case Western Reserve University

Department of Ophthalmology and Visual Sciences Cleveland, Ohio 44106

\begin{abstract}
Vision is initiated in photoreceptor cells of the retina by a set of biochemical events called phototransduction. These events occur via coordinated dynamic processes that include changes in secondary messenger concentrations, conformational changes and post-translational modifications of signaling proteins, and protein-protein interactions between signaling partners. A complete description of the orchestration of these dynamic processes is still unavailable. Described in this work is the first step in the development of tools combining fluorescent protein technology, Förster resonance energy transfer (FRET), and transgenic animals that have the potential to reveal important molecular insights about the dynamic processes occurring in photoreceptor cells. We characterize the fluorescent proteins SCFP3A and SYFP2 for use as a donor-acceptor pair in FRET assays, which will facilitate the visualization of dynamic processes in living cells. We also demonstrate the targeted expression of these fluorescent proteins to the rod photoreceptor cells of Xenopus laevis, and describe a general method for detecting FRET in these cells. The general approaches described here can address numerous types of questions related to phototransduction and photoreceptor biology by providing a platform to visualize dynamic processes in molecular detail within a native context. $\odot 2010$ Society of Photo-Optical Instrumentation Engineers. [DOI: 10.1117/1.3505023]
\end{abstract}

Keywords: Photoreceptor; phototransduction; Förster resonance energy transfer; fluorescent protein, G protein-coupled receptor; transgenic Xenopus laevis; retina; live cell.

Paper 10402R received Jul. 15, 2010; revised manuscript received Aug. 17, 2010; accepted for publication Sep. 13, 2010; published online Nov. 30, 2010.

\section{Introduction}

Vision is initiated upon photon capture in photoreceptor cells of the retina. Photon capture initiates a set of biochemical events called phototransduction, which culminate in the hyperpolarization of the cell. ${ }^{1,2}$ At the heart of any biological action lies the orchestration of several dynamic processes that contribute to the generation and regulation of the physiological response. In phototransduction, these dynamic processes include changes in concentration of the secondary messengers $\mathrm{Ca}^{2+}$ and cGMP, conformational changes and post-translational modifications of signaling and regulatory proteins, and protein-protein interactions that facilitate signal transduction. During signaling, these dynamic processes occur at specific locations and within a defined time frame. A complete and accurate molecular understanding of phototransduction is unavailable. To accurately understand the molecular orchestration underlying phototransduction, appropriate tools must be developed that allow for the investigation of the system in a native cellular context and with sufficient spatial and temporal resolution.

Fluorescent protein technology has significantly enhanced the ability to study biological processes within the context of an unperturbed living cell. ${ }^{3-5}$ Green fluorescent protein from

Address all Correspondence to: Paul S.-H. Park, Case Western Reserve University, Department of Ophthalmology and Visual Sciences, Cleveland, Ohio 44106. Tel: 216-368-2533; Fax: 216-368-3171. E-mail: paul.park@case.edu.
Aequorea victoria was the first fluorescent protein to be exploited for biochemistry and cell biology applications. ${ }^{3}$ Since then, the palette of fluorescent proteins has been greatly expanded to cover the entire visible spectrum. ${ }^{6-8}$ Proteins can be genetically modified to be in tandem with fluorescent proteins. These fusion proteins can be genetically expressed in cells, thereby allowing for noninvasive approaches to imaging molecules in living cells, tissues, and animals. Microscopy of fluorescent fusion proteins can provide both spatial and temporal details about molecular and cellular function.

The spatial resolution in conventional fluorescence microscopy is limited by the diffraction limit of light, and therefore dynamic processes such as protein-protein interactions and protein conformational changes, which occur over distances of less than $10 \mathrm{~nm}$, cannot be readily resolved. Förster resonance energy transfer (FRET) allows for the use of conventional fluorescence microscopy to detect events that occur over these short distances. FRET is a physical phenomenon where energy is transferred nonradiatively between donor and acceptor dipoles over distances in the range of 1 to $10 \mathrm{~nm}$ (Fig. 1) ${ }^{9-13}$ Fluorescent proteins with sufficient overlap between the emission spectrum of one and excitation spectrum of another can serve as a donor-acceptor pair for FRET. Cyan fluorescent protein (CFP) and yellow fluorescent protein (YFP), or their improved variants, are commonly used donor-acceptor fluorescent protein pairs for FRET studies. ${ }^{6,14}$ FRET between fluorescent

$1083-3668 / 2010 / 15(6) / 067001 / 9 / \$ 25.00$ (C) 2010 SPIE 


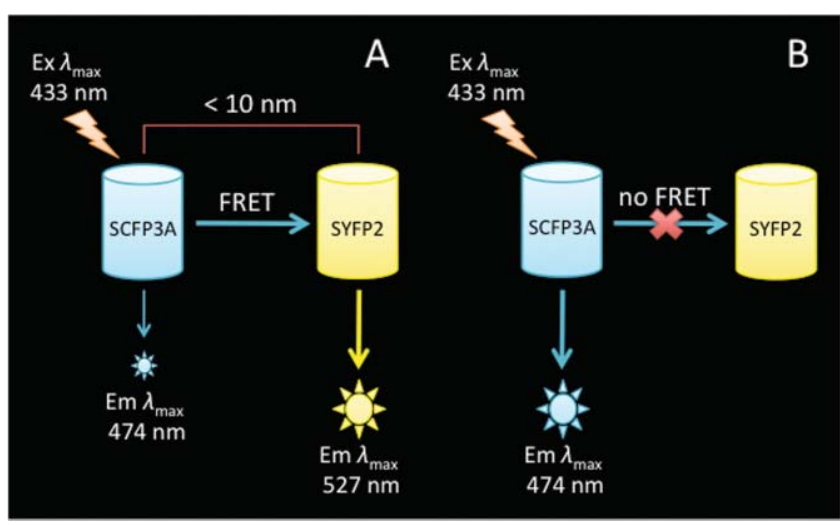

Fig. 1 Illustration highlighting the concept of FRET. (a) FRET occurs when SCFP3A and SYFP2 are within $10 \mathrm{~nm}$ of each other. FRET will result in the quenching of SCFP3A fluorescence and the sensitized emission of SYFP2 fluorescence upon excitation of SCFP3A. (b) When FRET does not occur, like when the two fluorescent proteins are greater than $10 \mathrm{~nm}$ apart or after photobleaching SYFP2, excitation of SCFP3A results only in its own emission without the sensitized signal from SYFP2. In acceptor-photobleaching assays, photobleaching of SYFP2 will result in an increased fluorescence emission from SCFP3A when FRET occurs between the two fluorescent proteins.

proteins can be used as sensitive biosensors, providing spatial and temporal information related to the dynamics of biological processes such as those occurring in phototransduction. ${ }^{11,15,16}$

Transgenesis in animals, such as mice, zebrafish, and frogs, has been widely used to understand photoreceptor biology. ${ }^{17}$ The rhodopsin promoter has been characterized in several animals, thereby facilitating the expression of transgene products specifically in the rod photoreceptor cells. ${ }^{18-20}$ The combination of fluorescent protein technology, FRET microscopy, and transgenesis in animals can provide detailed molecular views of the dynamic processes occurring in native photoreceptor cells, which is necessary to more accurately understand the molecular orchestration underlying phototransduction. We illustrate here the first steps in accomplishing these goals by transgenically expressing fluorescent proteins in rod photoreceptor cells of Xenopus laevis tadpoles, and establishing an imaging method based on commonly used wide-field microscopy to detect FRET between transgenically expressed fluorescent proteins in photoreceptor cells. Further development of these approaches will result in tools that will allow for the visualization of signaling events in live cells, tissues, and eventually animals.

\section{Materials and Methods}

\subsection{SCFP3A and SYFP2 Vectors}

Construction of the vectors pSCFP3A-C1, pSYFP2-C1, and pSYFP2-SCFP3A were described previously. ${ }^{21}$ The vectors pSCFP3A-N1 and pSYFP2-N1 were generated using the commercial vector pECFP-N1 (Clontech Laboratories, Mountain View, California). Sequences for SCFP3A and SYFP2 were amplified by polymerase chain reaction (PCR) using pSCFP3A$\mathrm{C} 1$ or pSYFP2-C1 as templates. The sequence for ECFP in pECFP-N1 was replaced by the sequence for either SCFP3A or SYFP2 to generate pSCFP3A-N1 and pSYFP2-N1, respectively. These expression vectors were used to transfect HEK293T cells.

The vectors pXOP-SCFP3A-N1, pXOP-SYFP2-N1, and pXOP-SYFP2-SCFP3A were constructed for use in the genera- tion of transgenic Xenopus laevis. These vectors have incorporated a Xenopus rhodopsin promoter and SV40 late polyadenylation sequence. The sequence of a 550-bp Xenopus rhodopsin promoter, characterized previously, ${ }^{19}$ was amplified by PCR to include NdeI, PmeI, and I-SceI sites at the $5^{\prime}$ end and an NheI site at the $3^{\prime}$ end. pXOP-SCFP3A-N1 and pXOP-SYFP2-N1 were generated using pSCFP3A-N1 and pSYFP2-N1. The CMV promoter sequence in pSCFP3A-N1 and pSYFP2-N1 was removed by digestion with the restriction enzymes NdeI and NheI (New England Biolabs, Ipswich, Massachusetts) and was replaced by the PCR-amplified Xenopus rhodopsin promoter sequence. The SV40 early polyadenylation signal sequence present in pSCFP3A-N1 and pSYFP2-N1 was removed by digestion with NotI and AfIII (New England Biolabs, Ipswich, Massachusetts) and replaced by the SV40 late polyadenylation signal sequence. The SV40 late polyadenylation sequence has been suggested to be more efficient in Xenopus laevis compared to the early sequence. ${ }^{22,23}$ The SV40 late polyadenylation signal sequence was amplified by PCR from the vector pCAT3-Basic (Promega, Madison, Wisconsin). The amplified product contained a NotI site at the $5^{\prime}$ end, and AfIII, PmeI, and I-SceI sites at the $3^{\prime}$ end. pXOP-SYFP2-SCFP3A was generated by replacing SYFP2 in $\mathrm{pXOP}-\mathrm{SYFP} 2-\mathrm{N} 1$ with the sequence for the fusion protein SYFP2-SCPF3A.

\subsection{Transfection in HEK293T Cells}

SCFP3A and SYFP2 were either individually expressed or coexpressed in HEK293T cells by transient transfection using Lipofectamine 2000 (Invitrogen, Carlsbad, California) and the vectors pSCFP3A-N1 and pSYFP2-N1. The SYFP2-SCFP3A fusion protein was expressed using the vector $\mathrm{pSYFP2-SCFP3A.}$ Cells were cultured in high glucose Dulbeccos Modified Eagles Medium (DMEM) (HyClone Laboratories, Logan, Utah) supplemented with $10 \%$ fetal bovine serum (HyClone Laboratories, Logan, Utah).

Cells used in spectrofluorometer assays were grown in 12-well plates. Cells from a single well were harvested 24-h post-transfection and resuspended in 3-mL Cellgro phosphate buffered saline (PBS) (catalog number 21-040, Mediatech, Manassas, Virginia). Cells that were fixed and imaged by microscopy were grown on $0.01 \%$ poly-L-lysine (Sigma-Aldrich, Saint Louis, Missouri) treated number 1.0 coverslips (Fisher Scientific, Pittsburgh, Pennsylvania) in a 12-well plate. 24 to 48-h post-transfection, cells were washed once with Cellgro PBS and then fixed in $4 \%$ paraformaldehyde for $10 \mathrm{~min}$. The fixed cells were washed three times in Cellgro PBS and sealed in $2 \%$ DABCO (Sigma-Aldrich, Saint Louis, Missouri). Cells used for live cell microscopy were grown on 35-mm poly-dlysine coated number 1.0 glass bottom dishes (MatTek, Ashland, Massachusetts). Transfected live cells were imaged at room temperature 24 to 48 -h post-transfection.

\subsection{Xenopus Laevis Transgenesis}

Xenopus laevis were purchased from Nasco (Fort Atkinson, Wisconsin). Transgenic tadpoles were generated using the sperm nuclear injection method, ${ }^{24}$ a simplified version of the restriction-enzyme mediated integration (REMI) method. ${ }^{25}$ pXOP-SCFP3A-N1, pXOP-SYFP2-N1, or 
pXOP-SYFP2-SCFP3A were digested with PmeI (New England Biolabs, Ipswich, Massachusetts) and the products separated on an agarose gel. The band containing DNA encoding the Xenopus rhodopsin promoter, transgene, and SV40 late polyadenylation sequence was isolated and the DNA purified using a QIAEX II gel extraction kit (Qiagen, Valencia, California). Injected embryos were incubated at $16{ }^{\circ} \mathrm{C}$ for 2 days and then transferred to room temperature. Tadpoles were screened 10 to 14 days postinjection and processed for histology or rod photoreceptor isolation.

For histology, tadpoles were first fixed in $4 \%$ paraformaldehyde in PBS (137-mM NaCl, 2.7-mM KCl, 4.3-mM $\mathrm{Na}_{2} \mathrm{HPO}_{4} \bullet 7 \mathrm{H}_{2} \mathrm{O}, 1.4-\mathrm{mM} \mathrm{KH}_{2} \mathrm{PO}_{4}, \mathrm{pH}$ 7.3). The tadpoles were then equilibrated in $20 \%$ sucrose (in PBS) and then in a 1:2 mixture of 20\% sucrose and Tissue-Tek OCT Compound (Sakura Finetek, Torrance, California). Each step was performed overnight at $4{ }^{\circ} \mathrm{C}$. The tadpoles were then embedded in OCT Compound by freezing in 2-methylbutane and sectioned at $14 \mu \mathrm{m}$ thickness. Sections were dried, rehydrated in PBS, stained with DAPI, and sealed in $2 \%$ DABCO prior to imaging.

For rod photoreceptor isolation, eyes were removed from tadpoles and the retina isolated. The retina was resuspended in amphibian Ringers buffer (100-mM NaCl, 2.5-mM KCl, 1.6-mM $\mathrm{MgCl}_{2}, 1-\mathrm{mM} \mathrm{CaCl}$, 5-mM HEPES, 5-mM glucose, $\mathrm{pH}$ 7.6) and vortexed for $5 \mathrm{~s}$. The suspension was centrifuged at $23 \times g$ for $5 \mathrm{~s}$ and the supernatant transferred to $35-\mathrm{mm}$ polyd-lysine coated number 1.0 glass bottom dishes (MatTek, Ashland, Massachusetts). The rod photoreceptor cells were allowed to settle to the bottom of the plate for $20 \mathrm{~min}$ before imaging by microscopy.

\subsection{Spectrofluorometer Characterization of Fluorescent Proteins}

Transfected HEK293T cells in Cellgro PBS were monitored for fluorescence on a FluoroMax-4 spectrofluorometer (Horiba Jobin Yvon, Edison, New Jersey). To determine the SCFP3A excitation spectrum, cells expressing SCFP3A alone were monitored for fluorescence at 550-nm emission wavelength (5-nm slit) across a range of excitation wavelengths (2-nm slit). To determine the SYFP2 excitation spectrum, cells expressing SYFP2 alone were monitored at an emission wavelength of $575 \mathrm{~nm}$ (5$\mathrm{nm}$ slit) across a range of excitation wavelengths (1-nm slit). The emission spectra of the two fluorescent proteins were determined by monitoring transfected cells at an excitation wavelength of $425 \mathrm{~nm}$ (2-nm slit) or $485 \mathrm{~nm}$ (1-nm slit) for SCFP3A or SYFP2, respectively, across a range of emission wavelengths (5-nm slit). The spectra obtained from untransfected HEK293T cells under each of the conditions were used for background subtraction. Cells coexpressing SCFP3A and SYFP2 or expressing the SYFP2-SCFP3A fusion protein were also monitored using the settings described before. Cells expressing SYFP2 alone monitored under SCFP3A emission spectrum settings or cells expressing SCFP3A alone monitored under SYFP2 emission spectrum settings were used for background subtraction.

\subsection{Förster Resonance Energy Transfer Microscopy}

Microscopy was performed on a Leica DMI3000B inverted microscope (Leica Microsystems, Bannockburn, Illinois). Images

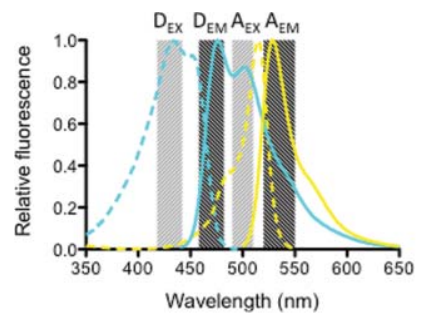

(a)

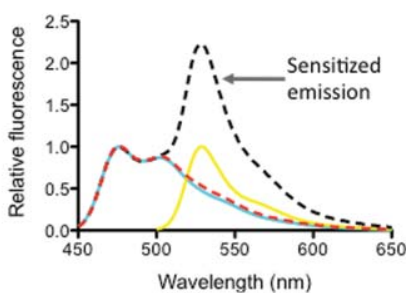

(b)
Fig. 2 Spectral characterization of SCFP3A and SYFP2 in live HEK293T cells. (a) The excitation spectra (dashed lines) and emission spectra (solid lines) of SCFP3A (cyan) and SYFP2 (yellow) are shown. The bandwidth of filters used in microscopy for excitation (EX) and emission detection (EM) of SCFP3A $\left(D_{\mathrm{EX}}, D_{\mathrm{EM}}\right)$ and SYFP2 $\left(A_{\mathrm{EX}}, A_{\mathrm{EM}}\right)$ is indicated by shaded bars. (b) The emission spectra of cells expressing the SYFP2-SCFP3A fusion protein (black) or coexpressing SCFP3A and SYFP2 (red) upon excitation at $425 \mathrm{~nm}$ are shown. The emission spectra of SCFP3A (cyan) and SYFP2 (yellow) from (a) are shown as a reference.

for FRET microscopy were obtained with a $63 \times / 1.4$-NA oil objective and recorded by a Rolera-MGi Plus EMCCD camera (QImaging, Surrey, British Columbia). The excitation light source was a Lambda LS xenon arc lamp (Sutter Instrument, Novato, California). The bandwidth of excitation and emission bandpass filters (Chroma Technology, Rockingham, Vermont) used in FRET assays is illustrated in Fig. 2(a). SCFP3A fluorescence was detected using the excitation and emission filters denoted as $D_{\mathrm{EX}}$ and $D_{\mathrm{EM}}$, respectively. SYFP2 fluorescence was detected using the excitation and emission filters denoted as $A_{\mathrm{EX}}$ and $A_{\mathrm{EM}}$, respectively. A single dichroic mirror was used with these filter sets, and both the excitation and emission filters were controlled using a Lambda 10-2 filter wheel system (Sutter Instrument, Novato, California). The FRET efficiency was estimated from two different methods: acceptor photobleaching and sensitized emission. SCFP3A served as the donor and SYFP2 served as the acceptor in FRET experiments.

Acceptor-photobleaching FRET was conducted only on HEK293T cells (Fig. 3). In acceptor-photobleaching FRET, the
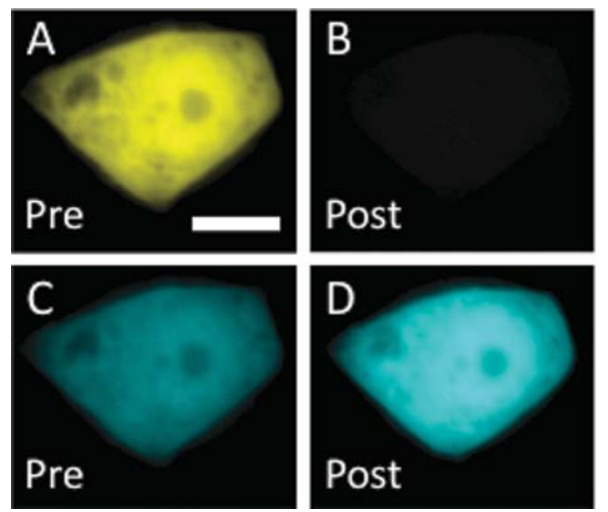

Fig. 3 Acceptor-photobleaching FRET in live HEK293T cells. A live cell expressing the SYFP2-SCFP3A fusion protein was imaged for (a) and (b) SYFP2 fluorescence $\left(A_{\mathrm{EX}}, A_{\mathrm{EM}}\right)$ and (c) and (d) SCFP3A fluorescence $\left(D_{\mathrm{EX}}, D_{\mathrm{EM}}\right)$ both before (pre) and after (post) photobleaching SYFP2. An increase in SCFP3A fluorescence after photobleaching SYFP2 is indicative of FRET [(c) versus $(\mathrm{d})]$. Scale bar $10 \mu \mathrm{m}$. 
Table 1 FRET efficiency values. Estimates of the FRET efficiency were computed as described in Sec. 2. The average FRET efficiency is shown with the standard deviation. The number of images used to compute these values is indicated in parentheses. The level of SYFP2 bleached in acceptor-photobleaching studies are as follows: HEK293T - fixed, SYFP2-SCFP3A, $89.15 \pm 3.54 \%$; HEK293T - fixed, SCPF3A + SYFP2, 86.36 $\pm 7.32 \%$; HEK293T - live, SYFP2-SCFP3A, $88.50 \pm 4.11 \%$; and HEK293T - live, SCFP3A + SYFP2, $86.98 \pm 4.67 \%$. The FRET efficiency was not determined (ND) by acceptor-photobleaching in photoreceptor cells.

FRET efficiency $(\%)$

\begin{tabular}{|c|c|c|c|c|}
\hline \multirow[t]{2}{*}{ Preparation } & \multicolumn{2}{|c|}{ SYFP2-SCFP3A fusion } & \multicolumn{2}{|c|}{ SCFP3A + SYFP2 coexpressed } \\
\hline & Sensitized emission & $\begin{array}{c}\text { Acceptor- } \\
\text { photobleaching }\end{array}$ & Sensitized emission & $\begin{array}{c}\text { Acceptor- } \\
\text { photobleaching }\end{array}$ \\
\hline HEK293T-fixed & $57.99 \pm 1.76(14)$ & $36.16 \pm 3.85(21)$ & $12.11 \pm 4.42(14)$ & $-0.41 \pm 2.49(15)$ \\
\hline HEK293T-live & $28.92 \pm 6.82(31)$ & $39.23 \pm 3.27(22)$ & $5.26 \pm 1.84(31)$ & $1.00 \pm 1.58(23)$ \\
\hline $\begin{array}{l}\text { Xenopus - retina } \\
\text { section }\end{array}$ & $49.85 \pm 6.94(11)$ & ND & $9.64 \pm 2.81(14)$ & ND \\
\hline $\begin{array}{l}\text { Xenopus - isolated } \\
\text { rods }\end{array}$ & $32.00 \pm 4.27(22)$ & ND & $2.27 \pm 1.85(14)$ & ND \\
\hline
\end{tabular}

average fluorescence intensity of SCFP3A was measured before and after photobleaching SYFP2. SYFP2 was photobleached by exposing fixed and live HEK293T cells to full power light using the $A_{\mathrm{EX}}$ filter for 5.5 and $3.5 \mathrm{~min}$, respectively. SYFP2 was bleached greater than $80 \%$ under these conditions (Table 1). The photobleaching conditions for SYFP2 did not cause appreciable bleaching of SCFP3A. The amount of SCFP3A bleached under SYFP2 photobleaching conditions is as follows: fixed HEK293T, $-0.29 \pm 1.81 \%(n=10)$; live HEK293T, -0.05 $\pm 1.52 \%(n=20)$. The FRET efficiency $(E)$ was computed according to the following equation: $E=1-\left(I_{D A} / I_{D}\right)$, where $I_{D A}$ and $I_{D}$ are the fluorescence intensities of the quenched donor and unquenched donor, respectively.

Sensitized emission FRET requires imaging of cells expressing SCFP3A or SYFP2 alone in addition to samples under investigation for FRET (e.g., SYFP2-SCFP3A fusion protein or coexpressed SCFP3A and SYFP2). Images were taken using SCPF3A fluorescence setting $\left(D_{\mathrm{EX}}, D_{\mathrm{EM}}\right)$, SYFP2 fluorescence setting $\left(A_{\mathrm{EX}}, A_{\mathrm{EM}}\right)$, and sensitized emission (FRET) setting $\left(D_{\mathrm{EX}}, A_{\mathrm{EM}}\right)$ (e.g., Fig. 4). The FRET image must be corrected for donor and acceptor spectral bleed-through signals to obtain the sensitized emission signal. Sensitized emission FRET was computed using pFRET data analysis software (W.M. Keck Center for Cellular Imaging, University of Virginia, Charlottesville, Virginia). ${ }^{26-28}$ The software removes donor and acceptor spectral bleed-through signals in the FRET image and corrects for variations in fluorescence intensity of the fluorophores. ${ }^{26}$ The FRET efficiency was calculated as follows:

$$
E=1-\left(\frac{\mathrm{I}_{\mathrm{DA}}+\mathrm{DSBT}}{\mathrm{I}_{\mathrm{DA}}+\mathrm{DSBT}+\mathrm{pFRET} \times\left(\mathrm{S}_{\mathrm{D}} / \mathrm{S}_{\mathrm{A}}\right) \times\left(\mathrm{Q}_{\mathrm{D}} / \mathrm{Q}_{\mathrm{A}}\right)}\right) .
$$

Donor and acceptor spectral bleed-through signals were determined from control images taken from cells expressing only SCFP3A or only SYFP2 [e.g., Figs. 4(a) through 4(f)]. The spectral bleed-through signals were subtracted from the FRET image

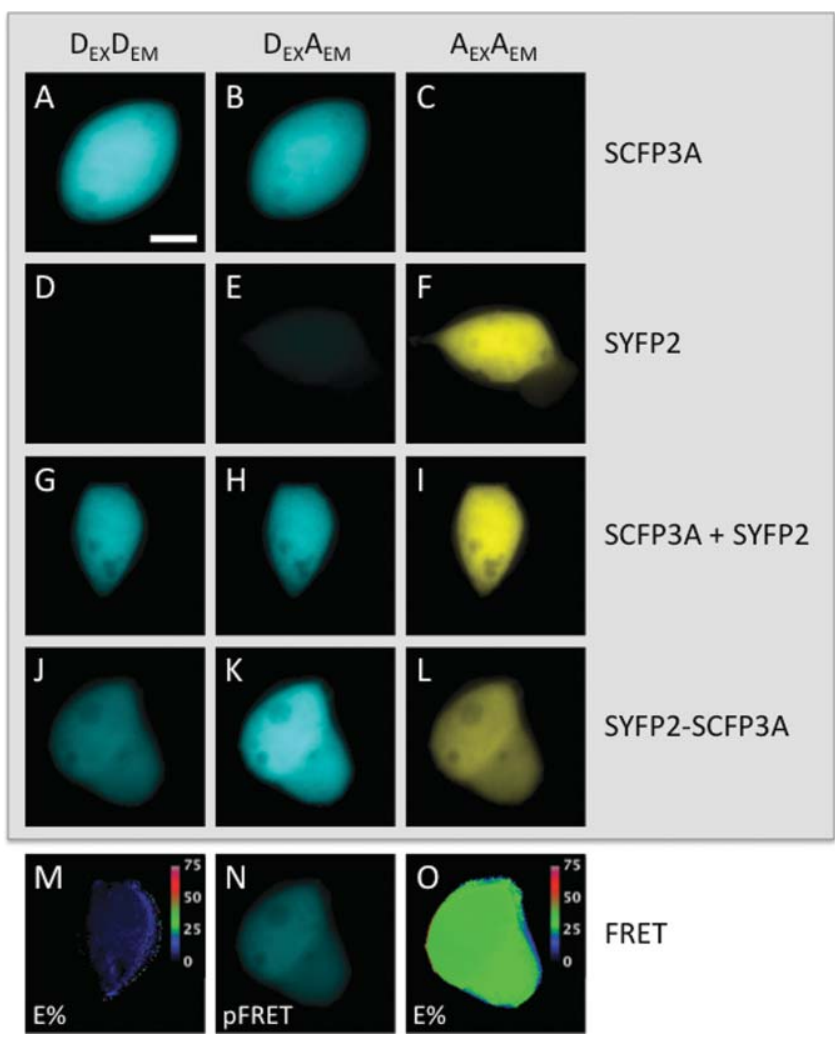

Fig. 4 Sensitized emission FRET in live HEK293T cells. Live cells (a), (b), and (c) expressing SCFP3A alone, (d), (e), and (f), expressing SYFP2 alone (g), (h), and (i) coexpressing SCFP3A and SYFP2, or (j), (k), and (l), expressing the SYFP2-SCFP3A fusion protein were imaged using the filter combinations indicated at the top of the figure. Control images obtained from cells expressing either SCFP3A or SYFP2 alone were used to determine the level of spectral bleed-through signals (b) and (e). The spectral bleed-through signals were removed from raw FRET images (h) and ( $k$ ) to obtain the corrected processed FRET image (pFRET). The pFRET image for a cell expressing the SYFP2-SCFP3A fusion protein is shown in $(\mathrm{n})$. Cells $(\mathrm{m})$ coexpressing SCFP3A and SYFP2 or (o) expressing the SYFP2-SCFP3A fusion protein are shown colored according to the computed FRET efficiency (E\%). Scale bar $10 \mu \mathrm{m}$. 
to obtain the pFRET signal. A correction was added to include the donor spectral bleed-through signal (DSBT) in the quenched donor to improve the accuracy of estimates of FRET efficiency. ${ }^{28}$ $S_{D}$ and $S_{A}$ are the spectral sensitivity of the camera for the donor and acceptor, respectively. These values were obtained from the manufacturer $\left(S_{D}=0.81 ; S_{A}=0.89\right) . Q_{D}$ and $Q_{A}$ are the quantum yield of SCFP3A and SYFP2, respectively ${ }^{21}\left(Q_{D}=0.56\right.$; $\left.Q_{A}=0.68\right)$.

\section{Results}

\subsection{Characterization of Förster Resonance Energy Transfer in HEK293T Cells}

The fluorescent proteins used in the current study are variants of the widely used CFP and YFP with improved brightness, protein folding, quantum yields, and FRET properties. ${ }^{21}$ The spectral properties of SCFP3A and SYFP2 were measured in live HEK293T cells expressing those fluorescent proteins [Fig. 2(a)]. The excitation and emission spectra of both proteins expressed in HEK293T cells were the same as those reported previously for the purified proteins. ${ }^{21}$ The large overlap between the emission spectrum of SCFP3A and excitation spectrum of SYFP2 make these two fluorescent proteins a suitable donor-acceptor pair for FRET.

The FRET capability between these two fluorescent proteins was first determined in a spectrofluorometer assay. When FRET occurs between the donor SCFP3A and acceptor SYFP2, excitation of the former results in the sensitized emission of fluorescence from the latter (Fig. 1). A fusion protein containing both SCFP3A and SYFP2 connected together with a flexible 12 amino acid linker sequence served as a positive FRET control in our studies. Excitation of cells expressing the SYFP2-SCFP3A fusion protein at SCFP3A excitation wavelength $(425 \mathrm{~nm})$ resulted in the sensitized emission of fluorescence from SYFP2 [Fig. 2(b), black]. In contrast, no sensitized emission was detected from SYFP2 when both SCFP3A and SYFP2 were coexpressed in HEK293T cells [Fig. 2(b), red]. Thus, no FRET occurs between SCFP3A and SYFP2 when they are coexpressed in the same cell. This negative control demonstrates that the two fluorescent proteins do not form aggregates, and that a positive FRET signal occurs only when they are in close proximity to each other.

We next established microscopy-based methods to detect FRET in both fixed and living HEK293T cells. The two microscopy approaches used were acceptor-photobleaching FRET and sensitized emission FRET. Acceptor-photobleaching FRET is based on the principle that SCFP3A fluorescence becomes quenched when FRET occurs between SCFP3A and SYFP2 [Fig. 1(a)]. When FRET between the two fluorescent proteins is disrupted by photobleaching SYFP2, the fluorescence of SCFP3A is no longer quenched [Fig. 1(b)]. Thus, the FRET efficiency can be estimated from the dequenching of SCFP3A fluorescence upon photobleaching SYFP2. The fluorescence of SCFP3A in cells expressing the fusion protein SYFP2-SCFP3A was monitored before and after photobleaching SYFP2 (Fig. 3). The photobleaching conditions for SYFP2 did not cause appreciable photobleaching of SCPF3A. The FRET efficiency computed from live and fixed HEK293T cells expressing the SYFP2-SCFP3A fusion protein was 39 and $36 \%$, respectively
(Table 1). No appreciable FRET was detected in HEK293T cells coexpressing SCFP3A and SYFP2 by acceptor-photobleaching FRET in both fixed and live cells.

Acceptor-photobleaching FRET is the more simple and straightforward microscopy method to obtain an estimate of the FRET efficiency compared to sensitized emission FRET, since there is no need to obtain control images and process images to remove contaminating spectral bleed-through signals. This approach, however, is not ideal to monitor dynamic processes in living cells by FRET, since the photobleaching time will not permit an adequate temporal window and only a single reading can be obtained because photobleaching of the acceptor molecule is irreversible. In addition, the intense light required for photobleaching can cause undesirable effects to the cell. Acceptorphotobleaching FRET is useful when monitoring static FRET and as a complementary approach to sensitized emission FRET to confirm observations.

Sensitized emission FRET can be used for time-lapse studies to monitor dynamic processes occurring in the cell. The temporal resolution will depend on the acquisition speed of the camera and the speed of filter exchange, which is in the millisecond range for the setup used here. FRET is monitored by the sensitized emission of SYFP2 upon excitation of SCFP3A [Fig. 1(a)]. The sensitized emission or FRET image is captured using the $D_{E X}$ and $A_{E M}$ filters [Fig. 2(a)]. The raw FRET image contains both the sensitized emission signal in addition to contaminating donor and acceptor spectral bleed-through signals [Figs. 4(b) and 4(e)]. Thus, the spectral bleed-through signals must be removed by collecting control images from cells expressing either SCFP3A or SYFP2 alone [Figs. 4(a) through 4(f)]. ${ }^{26}$

The computed FRET efficiency for the SYFP2-SCFP3A fusion protein from sensitized emission FRET was 58 and $29 \%$ for fixed and live HEK293T cells, respectively (Table 1). Thus, the FRET efficiency for the SYFP2-SCFP3A fusion protein is two-fold higher in fixed cells compared to live cells. Fixation or the mounting media does not appear to affect FRET itself, since the FRET efficiency computed from acceptorphotobleaching FRET data for fixed and live cells is comparable. The FRET efficiency computed for negative control cells coexpressing the two fluorescent proteins by sensitized emission FRET also was about two-fold higher for fixed cells compared to live cells (12 versus 5\%, Table 1). Thus, the higher FRET efficiency value obtained by sensitized emission FRET for fixed cells appears to be caused by errors in processing the raw FRET image to remove contaminating signals, which may be due to some effect from the fixative or mounting media. Fixation of cells has previously been shown to result in spurious FRET efficiency values, ${ }^{29,30}$ and therefore appropriate control studies like those conducted here must be performed.

Control studies should also be conducted for each different FRET methodology used, since the detection of FRET and the computation of FRET efficiencies can be different in each. A difference was observed in FRET efficiency estimates obtained by either acceptor-photobleaching or sensitized emission FRET in live HEK293T cells (39 versus 29\%, Table 1). The FRET efficiency for the SYFP2-SCPF3A fusion protein in live HeLa cells was determined previously by fluorescence lifetime imaging microscopy to yield a value of $27 \%,{ }^{21}$ which is similar to the value derived here in live HEK293T cells. The accuracy of FRET efficiency values for a given donor-acceptor pair is 
difficult to determine,${ }^{11}$ and without controls can be misleading. The control studies conducted in this study help to avoid unnecessary pitfalls, and clearly define a positive FRET signal from a false-positive FRET signal.

\subsection{Characterization of Förster Resonance Energy Transfer in Photoreceptor Cells}

Examination of SCFP3A and SYFP2 in HEK293T cells demonstrates that the spectral and FRET properties of these fluorescent proteins occur as expected. The ability to monitor FRET between SCFP3A and SYFP2 was next examined in rod photoreceptor cells of Xenopus laevis. Fluorescent proteins were expressed transgenically in Xenopus laevis tadpoles using the sperm nuclear injection method ${ }^{24}$ (Fig. 5). The fluorescent proteins were specifically targeted to the rod photoreceptor cells by utilizing a rhodopsin promoter previously characterized. ${ }^{19}$ Coexpression of SCFP3A and SYFP2 in rod photoreceptor cells was achieved by injecting embryos with both DNA constructs encoding one or the other fluorescent protein. Expression of protein using the rhodopsin promoter in Xenopus laevis has been shown to express in a mosaic pattern. ${ }^{31,32}$ However, even with a mosaic expression pattern, many of the photoreceptors were shown to express both transgene products in comparable quantities [Figs 5(d), 5(e), and 5(f)], which will be required in applications requiring the expression of two proteins.

Sensitized emission FRET was conducted in both fixed retina frozen sections and in isolated live photoreceptor cells (Fig. 6). Transgenic tadpoles were generated expressing either SCFP3A or SYFP2 alone to obtain control images for spectral bleedthrough subtraction. The FRET efficiency in fixed sections was $50 \%$ [Table 1, Fig. 6(c)], which is consistent with the high value obtained in fixed HEK293T cells. Likewise, the FRET efficiency for the SYFP2-SCFP3A fusion protein in live rod photoreceptor cells was similar to that obtained in live HEK293T cells with an estimated value of $32 \%$ [Table 1, Fig. 6(h)]. These studies
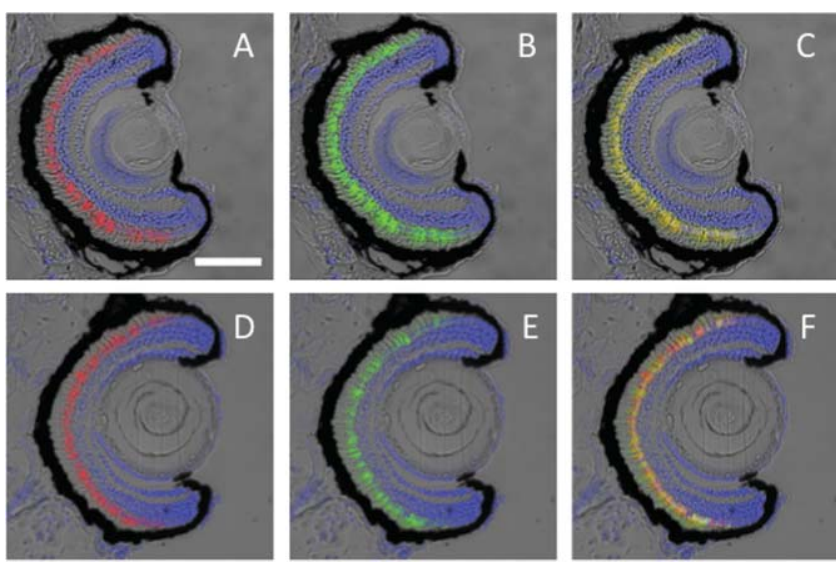

Fig. 5 Targeted expression of fluorescent proteins to rod photoreceptor cells. Transgenic Xenopus laevis tadpoles were generated expressing the SYFP2-SCFP3A fusion protein in (a), (b), and (c), or coexpressing SCFP3A and SYFP2 in (d), (e), and (f). Frozen retina sections were imaged for SCFP3A fluorescence (red, $D_{\mathrm{EX}}, D_{\mathrm{EM}}$ ) in (a) and (d) or SYFP2 fluorescence (green, $A_{\mathrm{EX}}, A_{\mathrm{EM}}$ ) in (b) and (e). Overlay of the SCFP3A and SYFP2 fluorescence signal is shown in (c) and (f). DAPI signal is shown in blue, and the fluorescence images are overlaid on top of the bright-field images. Scale bar $100 \mu \mathrm{m}$.
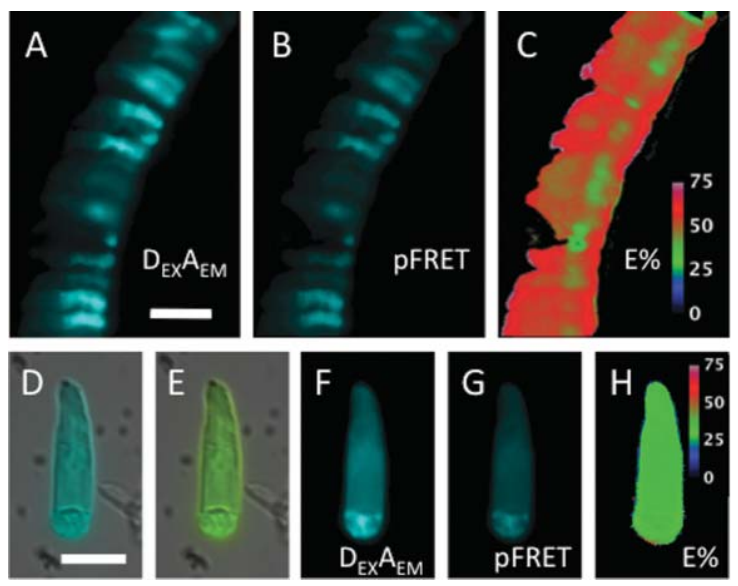

Fig. 6 FRET in photoreceptor cells. A frozen retina section in (a), (b), and (c) and isolated live rod photoreceptor cell in (d) through (h) from transgenic Xenopus laevis tadpoles expressing the SYFP2-SCFP3A fusion protein are shown. The raw FRET image in (a) and ( $\mathrm{f}$ ), corrected processed FRET image (pFRET) in (b) and ( $g$ ), and image colored according to the FRET efficiency $(E \%)$ in (c) and $(h)$ are shown. The pFRET image and FRET efficiency were determined using control images obtained from transgenic Xenopus laevis tadpoles expressing either SCFP3A or SYFP2 alone. SCFP3A fluorescence $\left(D_{\mathrm{EX}}, D_{\mathrm{EM}}\right)(\mathrm{d})$ and SYFP2 fluorescence $\left(A_{\mathrm{EX}}, A_{\mathrm{EM}}\right)(\mathrm{e})$ images are overlaid on top of the bright-field image from an isolated rod photoreceptor cell. Scale bars, are $25 \mu \mathrm{m}$ (a) and $10 \mu \mathrm{m}(\mathrm{d})$.

demonstrate the targeted expression of fluorescent proteins to rod photoreceptor cells, and the ability to detect FRET between SCFP3A and SYFP2 in both fixed retina frozen sections and isolated live photoreceptor cells. The methods highlighted here can be adapted to study specific aspects of photoreceptor biology.

\section{Discussion}

Phototransduction in rod photoreceptor cells has long served as a point of reference in understanding signaling pathways mediated by $\mathrm{G}$ protein-coupled receptors (GPCRs). ${ }^{1,33,34}$ Rhodopsin, the dim light receptor that initiates phototransduction in rod photoreceptor cells, is a prototypical GPCR. Several features of the phototransduction cascade are conserved across other GPCRmediated signaling pathways. ${ }^{35,36}$ FRET approaches, many utilizing CFP and YFP, have been used in a variety of ways to probe different facets of GPCR signaling systems, and have revealed many insights highlighting the need to reconsider some of the classical views of signal transduction. ${ }^{37-41}$

Taking cues from studies of GPCR-mediated signaling systems conducted in cell culture, one can envision several different ways that FRET can be utilized to study the dynamic processes in phototransduction. Dual incorporation of CFP and YFP tags into individual proteins can allow for the in-vivo monitoring of conformational changes that occur during the activation or deactivation of a signaling molecule (e.g., Refs. 42 and 43). FRET-based biosensors can provide spatial and temporal views of changes in concentration of the second messengers $\mathrm{Ca}^{2+}$ and cGMP, and dynamic post-translational modifications that regulate signaling events in live cells (e.g., Refs. 15, 44 and 45). Tagging of signaling proteins with FRET donor-acceptor pairs can allow the precise monitoring of protein-protein interactions 
to determine the nature of those interactions that facilitate the signaling process (e.g., Refs. 46-49).

The focus of the current study was to take the first step in developing FRET-based tools to study phototransduction under the native conditions of photoreceptor cells from the retina. This approach will advance what has already been accomplished for other GPCR systems by allowing for study in native tissue rather than cell culture systems. The characterization of the rhodopsin promoter in several vertebrate animals has allowed the targeted expression of proteins to the photoreceptor cells. ${ }^{18-20}$ The amenability of photoreceptor cells to this type of genetic manipulation provides an opportunity to study GPCRmediated cell signaling by FRET in a system derived from native tissue.

The rhodopsin promoter has been widely utilized to generate transgenic Xenopus laevis expressing fluorescent proteins or signaling proteins tagged with fluorescent proteins specifically in rod photoreceptor cells (e.g., Refs. 19, 31, 50-54). A similar approach has been used here to achieve the targeted expression of SCFP3A, SYFP2, and SCFP3A-SYFP2 fusion protein specifically to rod photoreceptor cells (Fig. 5). These soluble proteins localize in the inner segment of photoreceptor cells, and to a lesser degree in the outer segments. If greater outer segment localization is desired, fluorescent proteins can be modified genetically to contain an outer segment localization signal to achieve targeted expression to this region of the photoreceptor cell. $^{54-56}$

A microscopy-based method has been characterized in this study to detect FRET between the CFP and YFP variants SCFP3A and SYFP2, respectively, in native photoreceptor cells. Several different microscopy-based methods are available for FRET studies (e.g., Refs. 5, 9, and 14). The method described here is based on conventional wide-field microscopy. The instrumentation used in this study can achieve a temporal resolution in the millisecond range for time-lapse studies, and FRET provides a spatial resolution of less than $10 \mathrm{~nm}$. Thus, the spatial and temporal resolution provides an appropriate window in which to monitor various steps that occur during signaling. ${ }^{57}$

Photoreceptor cells can be studied in the intact retina or isolated, as we have done here. Isolated photoreceptor cells containing both outer and inner segments provide an excellent cellular model to study phototransduction, since their function is similar to that for photoreceptors still intact in the retina. ${ }^{58,59}$ To conduct FRET studies on photoreceptor cells in the intact retina, the methods presented here for wide-field microscopy can be adapted for confocal microscopy. Future advances may even allow for the study of photoreceptor cells in live animals, with improvements in optical methodology beyond what has been accomplished to date (e.g., Refs. 32 and 60).

It is inevitable that specific questions will require specific modifications. Since rhodopsin is a light sensitive receptor, special precautions will need to be made in sample preparation and data capture. Samples will need to be prepared under dim red light, and microscopes will need to be fitted with appropriate filters. For some applications, such as those looking at events occurring after photobleaching rhodopsin, the current donoracceptor FRET pair is sufficient. However, in other types of applications, it may be beneficial to consider alternate donoracceptor FRET pairs. For instance, applications that require rhodopsin to be protected from photobleaching can utilize a fluorescent protein with a shorter excitation wavelength profile to serve as the donor molecule, such as blue fluorescent protein (e.g., Ref. 61). The combination of technologies outlined in this study form the basic platform for FRET approaches to investigate signaling events in phototransduction and, with the appropriate modifications, will provide necessary tools to move us forward in obtaining accurate views of the molecular orchestration occurring in photoreceptor cells.

\section{Acknowledgments}

We would like to thank Dawn Smith for culturing and transfecting HEK293T cells, Catherine Doller for sectioning frozen tadpoles, Ammasi Periasamy for providing pFRET software, Theodorus W. J. Gadella for providing the vectors pSCFP3A-C1, SYFP2-C1, and pSYFP2-SCFP3A, Barry E. Knox for providing the vector pXOP-XOP, and Heithem El-Hodiri and Yi Pan for their gracious help in the early part of this study and for showing us the transgenesis procedure. This study was supported by grants from the National Institutes of Health (R00EY018085 and P30EY11373), Research to Prevent Blindness, and the Ohio Lions Eye Research Foundation.

\section{References}

1. V. Y. Arshavsky, T. D. Lamb, and E. N. Pugh, Jr., "G proteins and phototransduction," Annu. Rev. Physiol. 64, 153-187 (2002).

2. K. W. Yau and R. C. Hardie, "Phototransduction motifs and variations," Cell 139(2), 246-264 (2009).

3. R. Y. Tsien, "The green fluorescent protein," Annu. Rev. Biochem. 67, 509-544 (1998).

4. R. Y. Tsien, and A. Miyawaki, "Seeing the machinery of live cells," Science 280(5371), 1954-1955 (1998).

5. R. B. Sekar, and A. Periasamy, "Fluorescence resonance energy transfer (FRET) microscopy imaging of live cell protein localizations," J. Cell. Biol. 160(5), 629-633 (2003).

6. R. N. Day, and M. W. Davidson, "The fluorescent protein palette: tools for cellular imaging," Chem. Soc. Rev. 38(10), 2887-2921 (2009).

7. N. C. Shaner, P. A. Steinbach, and R. Y. Tsien, "A guide to choosing fluorescent proteins," Nat. Meth. 2(12), 905-909 (2005).

8. N. C. Shaner, G. H. Patterson, and M. W. Davidson, "Advances in fluorescent protein technology," J. Cell. Sci. 120(Pt 24), 4247-4260 (2007).

9. E. A. Jares-Erijman, and T. M. Jovin, "FRET imaging," Nat. Biotechnol. 21(11), 1387-1395 (2003).

10. P. Wu, and L. Brand, "Resonance energy transfer: methods and applications," Anal. Biochem. 218(1), 1-13 (1994).

11. S. S. Vogel, C. Thaler, and S. V. Koushik, "Fanciful FRET," Sci. STKE 2006(331), re2 (2006).

12. Z. Gryczynski, I. Gryczynski, and J. R. Lakowicz, "Basics of Fluorescence and FRET," in Molecular Imaging: FRET Microscopy and Spectroscopy, A. Periasamy, and R. N. Day, Eds., pp 21-56. Oxford University Press, New York (2005).

13. J. R. Lakowicz, Principles of Fluorescence Spectroscopy. 3rd eds., Springer Science + Business Media, LLC, New York (2006).

14. D. W. Piston, and G. J. Kremers, "Fluorescent protein FRET: the good, the bad and the ugly," Trends Biochem. Sci. 32(9), 407-414 (2007).

15. N. N. Aye-Han, and Q. Ni J. Zhang, "Fluorescent biosensors for real-time tracking of post-translational modification dynamics," Curr. Opin. Chem. Biol. 13(4), 392-397 (2009).

16. H. J. Carlson, and R. E. Campbell, "Genetically encoded FRET-based biosensors for multiparameter fluorescence imaging," Curr. Opin. Biotechnol. 20(1), 19-27 (2009). 
17. C. L. Makino, X. H. Wen, and J. Lem, "Piecing together the timetable for visual transduction with transgenic animals," Curr. Opin. Neurobiol. 13(4), 404-412 (2003).

18. J. Lem, M. L. Applebury, J. D. Falk, J. G. Flannery, and M. I. Simon, "Tissue-specific and developmental regulation of rod opsin chimeric genes in transgenic mice," Neuron 6(2), 201-210 (1991).

19. S. S. Mani, S. Batni, L. Whitaker, S. Chen, G. Engbretson, and B. E. Knox, "Xenopus rhodopsin promoter. Identification of immediate upstream sequences necessary for high level, rod-specific transcription," J. Biol. Chem. 276(39), 36557-36565 (2001).

20. B. N. Kennedy, T. S. Vihtelic, L. Checkley, K. T. Vaughan, and D. R. Hyde, "Isolation of a zebrafish rod opsin promoter to generate a transgenic zebrafish line expressing enhanced green fluorescent protein in rod photoreceptors," J. Biol. Chem. 276(17), 14037-14043 (2001).

21. G. J. Kremers, J. Goedhart, E. B. van Munster, and T. W. Gadella, Jr., "Cyan and yellow super fluorescent proteins with improved brightness, protein folding, and FRET Forster radius," Biochem. 45(21), 6570-6580 (2006).

22. S. Carswell and J. C. Alwine, "Efficiency of utilization of the simian virus 40 late polyadenylation site: effects of upstream sequences," $\mathrm{Mol}$. Cell. Biol. 9(10), 4248-4258 (1989).

23. K. M. Kwan, E. Fujimoto, C. Grabher, B. D. Mangum, M. E. Hardy, D. S. Campbell, J. M. Parant, H. J. Yost, J. P. Kanki, and C. B. Chien, "The Tol2kit: a multisite gateway-based construction kit for Tol2 transposon transgenesis constructs," Dev. Dyn. 236(11), 3088-3099 (2007).

24. S. J. Smith, L. Fairclough, B. V. Latinkic, D. B. Sparrow, and T. J. Mohun, "Xenopus laevis transgenesis by sperm nuclear injection," Nat. Protoc. 1(5), 2195-2203 (2006).

25. E. Amaya, and K. L. Kroll, "A method for generating transgenic frog embryos,” Meth. Mol. Biol. 97, 393-414 (1999).

26. Y. Chen, M. Elangovan, and A. Periasamy, "FRET data analysis: the algorithm," in Molecular Imaging: FRET Microscopy and Spectroscopy, A. Periasamy, and R. N. Day, Eds., pp 126-145, Oxford University Press, New York (2005).

27. M. Elangovan, H. Wallrabe, Y. Chen, R. N. Day, M. Barroso, and A. Periasamy, "Characterization of one- and two-photon excitation fluorescence resonance energy transfer microscopy," Meth. 29(1), 58-73 (2003).

28. Y. Sun, and A. Periasamy, "Additional correction for energy transfer efficiency calculation in filter-based Forster resonance energy transfer microscopy for more accurate results," J. Biomed. Opt. 15(2), 020513 (2010).

29. M. Anikovsky, L. Dale, S. Ferguson, and N. Petersen, "Resonance energy transfer in cells: a new look at fixation effect and receptor aggregation on cell membrane," Biophys. J. 95(3), 1349-1359 (2008).

30. S. Rodighiero, C. Bazzini, M. Ritter, J. Furst, G. Botta, G. Meyer, and M. Paulmichl, "Fixation, mounting and sealing with nail polish of cell specimens lead to incorrect FRET measurements using acceptor photobleaching," Cell. Physiol. Biochem. 21(5-6), 489-498 (2008).

31. S. Jin, T. D. McKee, and D. D. Oprian, "An improved rhodopsin/EGFP fusion protein for use in the generation of transgenic Xenopus laevis," FEBS Lett. 542(1-3), 142-146 (2003).

32. O. L. Moritz, B. M. Tam, B. E. Knox, and D. S. Papermaster, "Fluorescent photoreceptors of transgenic Xenopus laevis imaged in vivo by two microscopy techniques," Invest. Ophthalmol. Vis. Sci. 40(13), 3276-3280 (1999).

33. K. D. Ridge, N. G. Abdulaev, M. Sousa, and K. Palczewski, "Phototransduction: crystal clear," Trends Biochem. Sci. 28(9), 479-487 (2003).

34. P. S. H. Park, S. Filipek, J. W. Wells, and K. Palczewski, "Oligomerization of $\mathrm{G}$ protein-coupled receptors: past, present, and future," Biochem. 43(50), 15643-15656 (2004).

35. G. L. Wheeler, and M. W. Bitensky, "A light-activated GTPase in vertebrate photoreceptors: regulation of light-activated cyclic GMP phosphodiesterase," Proc.Natl.Acad.Sci.USA 74(10), $4238-4242$ (1977).

36. M. W. Bitensky, M. A. Wheeler, M. M. Rasenick, A. Yamazaki, P. J. Stein, K. R. Halliday, and G. L. Wheeler, "Functional exchange of components between light-activated photoreceptor phosphodiesterase and hormone-activated adenylate cyclase systems," Proce. Nat. Acad. Sci. USA 79(11), 3408-3412 (1982).

37. C. Gales, R. V. Rebois, M. Hogue, P. Trieu, A. Breit, T. E. Hebert, and M. Bouvier, "Real-time monitoring of receptor and G-protein interactions in living cells," Nat. Meth. 2(3), 177-184 (2005).

38. T. E. Hebert, C. Gales, and R. V. Rebois, "Detecting and imaging protein-protein interactions during $\mathrm{G}$ protein-mediated signal transduction in vivo and in situ by using fluorescence-based techniques," Cell. Biochem. Biophys. 45(1), 85-109 (2006).

39. T. Balla, "Green light to illuminate signal transduction events," Trends Cell. Biol. 19(11), 575-586 (2009).

40. M. J. Lohse, M. Bunemann, C. Hoffmann, J. P. Vilardaga, and V. O. Nikolaev, "Monitoring receptor signaling by intramolecular FRET," Curr. Opin. Pharmacol. 7(5), 547-553 (2007).

41. J. P. Vilardaga, M. Bunemann, T. N. Feinstein, N. Lambert, V. O. Nikolaev, S. Engelhardt, M. J. Lohse, and C. Hoffmann, "GPCR and G proteins: drug efficacy and activation in live cells," Mol. Endocrinol. 23(5), 590-599 (2009).

42. P. G. Charest, S. Terrillon, and M. Bouvier, "Monitoring agonistpromoted conformational changes of beta-arrestin in living cells by intramolecular BRET," EMBO Rep. 6(4), 334-340 (2005).

43. J. P. Vilardaga, M. Bunemann, C. Krasel, M. Castro, and M. J. Lohse, "Measurement of the millisecond activation switch of G proteincoupled receptors in living cells," Nat. Biotechnol. 21(7), 807-812 (2003).

44. A. Miyawaki, J. Llopis, R. Heim, J. M. McCaffery, J. A. Adams, M. Ikura, and R. Y. Tsien, "Fluorescent indicators for $\mathrm{Ca} 2+$ based on green fluorescent proteins and calmodulin," Nature 388(6645), 882-887 (1997).

45. V. O. Nikolaev, S. Gambaryan, and M. J. Lohse, "Fluorescent sensors for rapid monitoring of intracellular cGMP," Nat. Meth. 3(1), 23-25 (2006).

46. J. B. Jensen, J. S. Lyssand, C. Hague, and B. Hille, "Fluorescence changes reveal kinetic steps of muscarinic receptor-mediated modulation of phosphoinositides and Kv7.2/7.3 K + channels," J. Gen. Physiol. 133(4), 347-359 (2009).

47. B. Hollins, S. Kuravi, G. J. Digby, and N. A. Lambert, "The c-terminus of GRK3 indicates rapid dissociation of G protein heterotrimers," Cell. Signal. 21(6), 1015-1021 (2009).

48. R. V. Rebois, M. Robitaille, C. Gales, D. J. Dupre, A. Baragli, P. Trieu, N. Ethier, M. Bouvier, and T. E. Hebert, "Heterotrimeric G proteins form stable complexes with adenylyl cyclase and Kir3.1 channels in living cells," J. Cell. Sci. 119(Pt 13), 2807-2818 (2006).

49. C. Gales, J. J. Van Durm, S. Schaak, S. Pontier, Y. Percherancier, M. Audet, H. Paris, and M. Bouvier, "Probing the activation-promoted structural rearrangements in preassembled receptor-G protein complexes," Nat. Struct. Mol. Biol. 13(9), 778-786 (2006).

50. J. J. Peterson, B. M. Tam, O. L. Moritz, C. L. Shelamer, D. R. Dugger, J. H. McDowell, P. A. Hargrave, D. S. Papermaster, and W. C. Smith, "Arrestin migrates in photoreceptors in response to light: a study of arrestin localization using an arrestin-GFP fusion protein in transgenic frogs," Exp. Eye. Res. 76(5), 553-563 (2003).

51. Q. Wang, X. Zhang, L. Zhang, F. He, G. Zhang, M. Jamrich, and T. G. Wensel, "Activation-dependent hindrance of photoreceptor G protein diffusion by lipid microdomains," J. Biol. Chem. 283(44), 30015-30024 (2008).

52. J. A. Peet, A. Bragin, P. D. Calvert, S. S. Nikonov, S. Mani, X. Zhao, J. C. Besharse, E. A. Pierce, B. E. Knox, and E. N. Pugh, Jr., "Quantification of the cytoplasmic spaces of living cells with EGFP reveals arrestin-EGFP to be in disequilibrium in dark adapted rod photoreceptors," J. Cell. Sci. 117(Pt 14), 3049-3059 (2004).

53. O. L. Moritz, B. M. Tam, D. S. Papermaster, and T. Nakayama, "A functional rhodopsin-green fluorescent protein fusion protein localizes correctly in transgenic Xenopus laevis retinal rods and is expressed in a time-dependent pattern," J. Biol. Chem. 276(30), 28242-28251 (2001).

54. S. A. Baker, M. Haeri, P. Yoo, S. M. Gospe, 3rd, N. P. Skiba, B. E. Knox, and V. Y. Arshavsky, "The outer segment serves as a default destination for the trafficking of membrane proteins in photoreceptors," J. Cell. Biol. 183(3), 485-498 (2008). 
Hovan, Howell, and Park: Förster resonance energy transfer as a tool to study photoreceptor biology

55. W. Luo, N. Marsh-Armstrong, A. Rattner, and J. Nathans, "An outer segment localization signal at the $\mathrm{C}$ terminus of the photoreceptorspecific retinol dehydrogenase," J. Neurosci. 24(11), 2623-2632 (2004).

56. B. M. Tam, O. L. Moritz, L. B. Hurd, and D. S. Papermaster, "Identification of an outer segment targeting signal in the $\mathrm{COOH}$ terminus of rhodopsin using transgenic Xenopus laevis," J. Cell. Biol. 151(7), 1369-1380 (2000).

57. M. J. Lohse, V. O. Nikolaev, P. Hein, C. Hoffmann, J. P. Vilardaga, and M. Bunemann, "Optical techniques to analyze real-time activation and signaling of G-protein-coupled receptors," Trends Pharmacol. Sci. 29(3), 159-165 (2008).
58. M. S. Biernbaum, and M. D. Bownds, "Frog rod outer segments with attached inner segment ellipsoids as an in vitro model for photoreceptors on the retina," J. Gen. Physiol. 85(1), 83-105 (1985).

59. C. Chen, E. Tsina, M. C. Cornwall, R. K. Crouch, S. Vijayaraghavan, and Y. Koutalos, "Reduction of all-trans retinal to all-trans retinol in the outer segments of frog and mouse rod photoreceptors," Biophys. J. 88(3), 2278-2287 (2005).

60. Y. Imanishi, K. H. Lodowski, and Y. Koutalos, "Two-photon microscopy: shedding light on the chemistry of vision," Biochem. 46(34), 9674-9684 (2007).

61. B. A. Pollok, and R. Heim, "Using GFP in FRET-based applications," Trends Cell. Biol. 9(2), 57-60 (1999). 